\title{
STATISTICAL STUDIES OF DURATION OF LOW-POWER SOLAR FLARES
}

\author{
A.V. Borovik \\ Institute of Solar-Terrestrial Physics SB RAS, \\ Irkutsk, Russia,aborovik@iszf.irk.ru
}

\author{
A.A. Zhdanov \\ Institute of Solar-Terrestrial Physics SB RAS, \\ Irkutsk, Russia, kick.out@mail.ru
}

\begin{abstract}
This paper is a sequel to papers dealing with time parameters of solar flares in the $\mathrm{H} \alpha$ line. Using data from the international flare patrol for 1972 2010, we have determined the mean duration of flares of different importance and classes of area. We have established that $94.6 \%$ of flares last more than $60 \mathrm{~min}$. The duration of $90 \%$ of flares with $\bar{t}>60 \mathrm{~min}$ is $2.1-3.3$ hrs. In rare cases, flares can last about 12 hrs. The duration of powerful solar flares does not exceed $8.3 \mathrm{hrs}$.
\end{abstract}

We have found that the duration of solar flares depends on features of their development. Flares with one brilliant point in the flare region have the shortest duration; two-ribbon flares and flares exhibiting several intensity maxima have the longest duration. We have confirmed that the duration of flares increases with increasing classes of area and brightness.

Keywords: solar activity, solar flares.

\section{INTRODUCTION}

Time parameters of solar flares were studied in detail during the formation of the international flare patrol. The first results obtained from relatively small data samples have shown that solar flares can last from a few minutes to 12 hours or more [Smith H., Smith E., 1966].

By now, the international solar patrol has collected extensive statistical data on solar flares. This makes it possible to conduct additional studies, to specify time parameters of solar flares. In recent decades, this issue has been repeatedly addressed [Temmer et al., 2001; Giersch, 2013; Potzi et al., 2014], but no detailed analysis has been made so far.

The duration of solar flares is composed of two characteristic intervals: initial (flash) phase and main phase. The main phase has been examined in detail in [Borovik, Zhdanov, 2017a]. This paper studies the total duration of solar flares. Particular attention, as in previous works, is given to low-power flares with an area of less than 2 sq. deg.

\section{DATABASE AND ITS ANALYSIS}

An electronic database of solar flares has been created from Solar Geophysical Data (SGD) and Quarterly Bulletin on Solar Activity over the period from 1972 to 2010. It includes parameters of 123801 flares, 110778 of which are low-power, 11280 are class 1, and 1743 are class 2-4 flares. In source catalogs, software-based methods have been used to correct errors and eliminate duplication of events. The cases when stations did not report on the class of brightness, area, time of onset, maximum, and end of a flare, etc. are taken into account.

International flare patrol data are heterogeneous [Altyntsev et al., 1982]. Durations of flares in group reports from different stations often differ. To estimate variance in the groups, we have determined standard deviations of flare duration:

$$
\sigma=\sqrt{\frac{1}{n-1}} \sum_{i=1}^{n}\left(x_{i}-\bar{x}\right)^{2},
$$

where $n$ is the number of reports on a given flare from different stations. The standard deviations were then averaged over corresponding importance and classes of area (Table 1, Figure 1). From these groups we excluded summarized data and data obtained visually and under poor observation conditions, as well as those with letter qualifiers (D, E, $U$ and *). The number of the analyzed groups $N_{\mathrm{gr}}$ is given in the second column of Table 1 .

The results show that with increasing class of area and importance of flares, $\bar{\sigma}$ gradually increases from 5.2 to $17.8 \mathrm{~min}$.

To reduce the variance, large data samples or observations from an individual station are usually used. We have employed both the approaches.

Table 1

\begin{tabular}{|l|r|r|}
\hline & \multicolumn{1}{|c|}{$N_{\mathrm{gr}}$} & $\bar{\sigma}$ \\
\hline SF & 8433 & 5.2 \\
\hline SN & 2991 & 6.5 \\
\hline SB & 699 & 6.7 \\
\hline $1 \mathrm{~F}$ & 161 & 7.0 \\
\hline $1 \mathrm{~N}$ & 469 & 9.8 \\
\hline $1 \mathrm{~B}$ & 395 & 10.2 \\
\hline$(2-4) \mathrm{F}$ & 13 & 10.7 \\
\hline$(2-4) \mathrm{N}$ & 65 & 10.6 \\
\hline$(2-4) \mathrm{B}$ & 161 & 17.8 \\
\hline $\mathrm{S}$ & 16352 & 6.2 \\
\hline 1 & 1697 & 10.5 \\
\hline $2-4$ & 359 & 16.4 \\
\hline
\end{tabular}




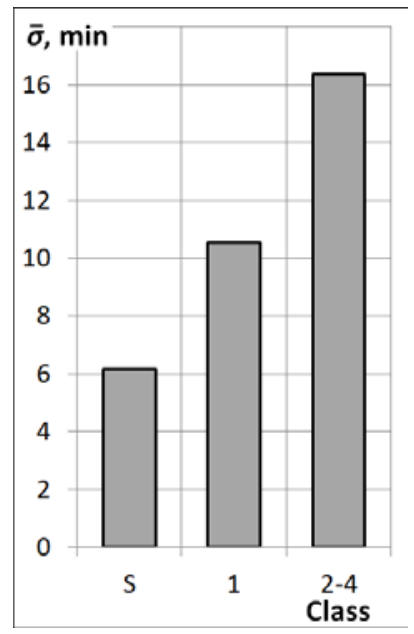

Figure 1. $\bar{\sigma}$ as a function of class of area

\section{DURATION OF SOLAR FLARES ACCORDING TO DATA FROM ALL STATIONS}

As in [Borovik, Zhdanov, 2017a], we impose the same restrictions on the database in assessing time parameters of solar flares as in estimating variance in groups (see the previous section). Since for small flares on the edge of the solar disk the data are not as reliable as for flares near its center, class $\mathrm{S}$ and 1 flares with a longitude $>65^{\circ}$ are excluded from the database. Such a restriction did not apply to large flares. After applying the above restrictions, in the groups we use flare data from the station that has the most reports in the database.

As a result of the selection, the total number of flares is 85983 . Their distribution by duration (in percent of the total number of flares with an increment of $1 \mathrm{~min}$ ) is shown in Figure 2. The vertical dashed line indicates the position of the median. The tail of the distribution is scaled up (Figure 2, right axis).

The resulting distribution is rather asymmetric and extended. Duration of some flares exceeds $360 \mathrm{~min}$. The distribution range is therefore limited to $322 \mathrm{~min}$ the time when the distribution continuity breaks down. The unaccounted flares (15) are $0.02 \%$. Statistical parameters of flares are then estimated only within this range.

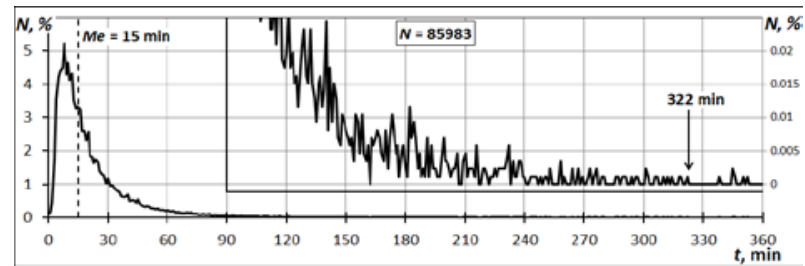

Figure 2. Duration distribution of solar flares

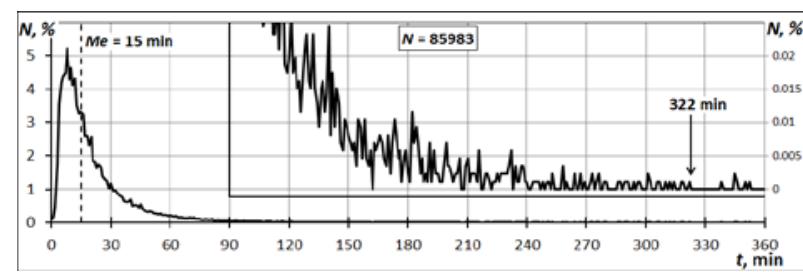

Figure 2. Duration distribution of solar flares
Figures 3-5 show the duration distribution of flares for individual classes of area and importance. Large flares, in order to give them a larger statistical weight, are combined in group 2-4.

All distributions have a pronounced positive asymmetry and one pronounced maximum, followed by an extended decrease. Table 2 lists the number of flares $(N)$, the mean duration $(\bar{t})$ with a confidence interval $(\alpha)$, modal $(\mathrm{Mo})$ and median $(\mathrm{Me})$ distribution parameters, time intervals for $90 \%$ of flares $(\Delta t)$.

Table 2

\begin{tabular}{|l|r|c|r|r|r|}
\hline & \multicolumn{1}{|c|}{$N$} & $\bar{t} \pm \alpha$ & $M o$ & $M e$ & $\Delta t$ \\
\hline SF & 55799 & $17.4 \pm 0.1$ & 8 & 13 & $1-35$ \\
\hline SN & 18538 & $22.1 \pm 0.3$ & 10 & 17 & $1-43$ \\
\hline SB & 3867 & $25.9 \pm 0.7$ & 12 & 19 & $1-53$ \\
\hline 1F & 1959 & $40.3 \pm 1.4$ & 17 & 33 & $1-79$ \\
\hline 1N & 2912 & $44.0 \pm 1.3$ & 22 & 35 & $1-85$ \\
\hline 1B & 1590 & $51.5 \pm 1.8$ & 31 & 42 & $1-99$ \\
\hline$(2-4) \mathrm{F}$ & 172 & $57.1 \pm 6.7$ & 26 & 45 & $1-113$ \\
\hline$(2-4) \mathrm{N}$ & 460 & $69.3 \pm 4.5$ & 41 & 55 & $1-137$ \\
\hline$(2-4) \mathrm{B}$ & 671 & $85.5 \pm 4.5$ & 49 & 69 & $1-170$ \\
\hline S & 78204 & $18.9 \pm 0.1$ & 8 & 14 & $1-38$ \\
\hline 1 & 6461 & $44.7 \pm 0.8$ & 20 & 36 & $1-87$ \\
\hline $2-4$ & 1303 & $76.0 \pm 3.0$ & 44 & 61 & $1-149$ \\
\hline$\sum$ & 85968 & $21.7 \pm 0.2$ & 8 & 15 & $1-45$ \\
\hline
\end{tabular}
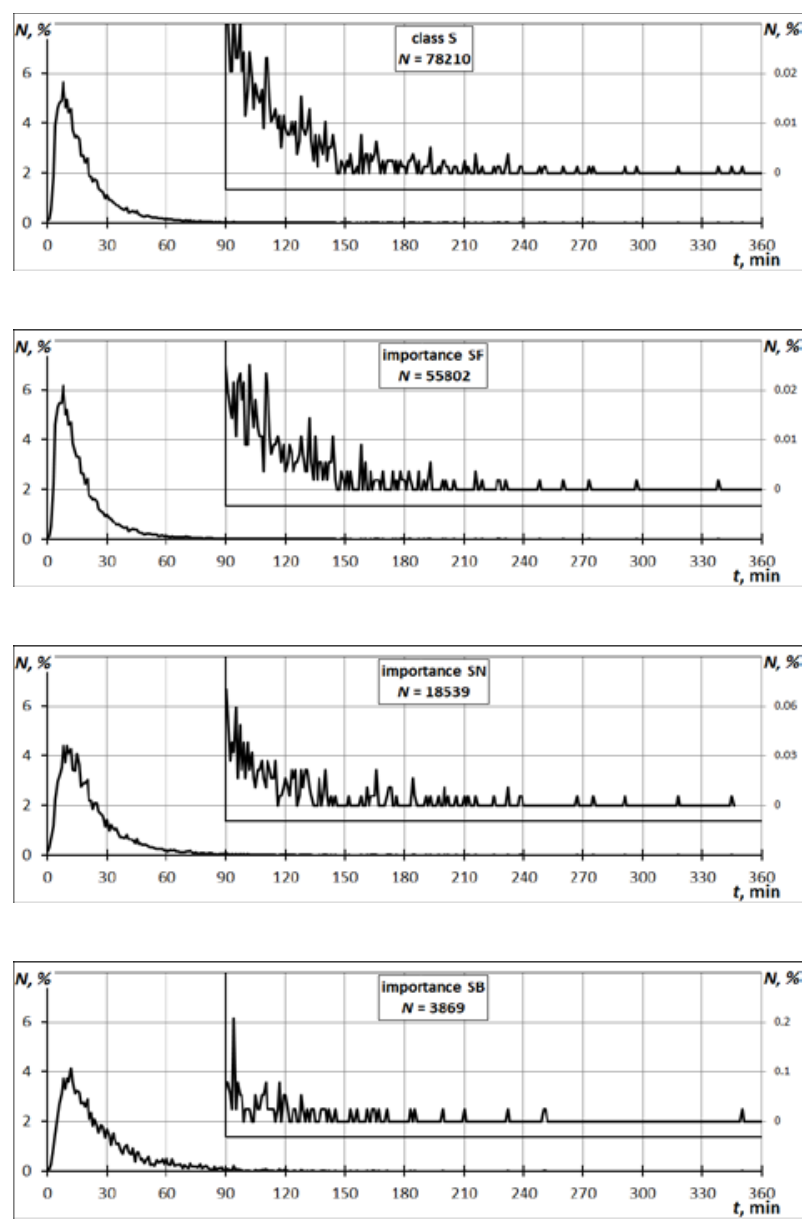

Figure 3. Duration distribution of class S flares 


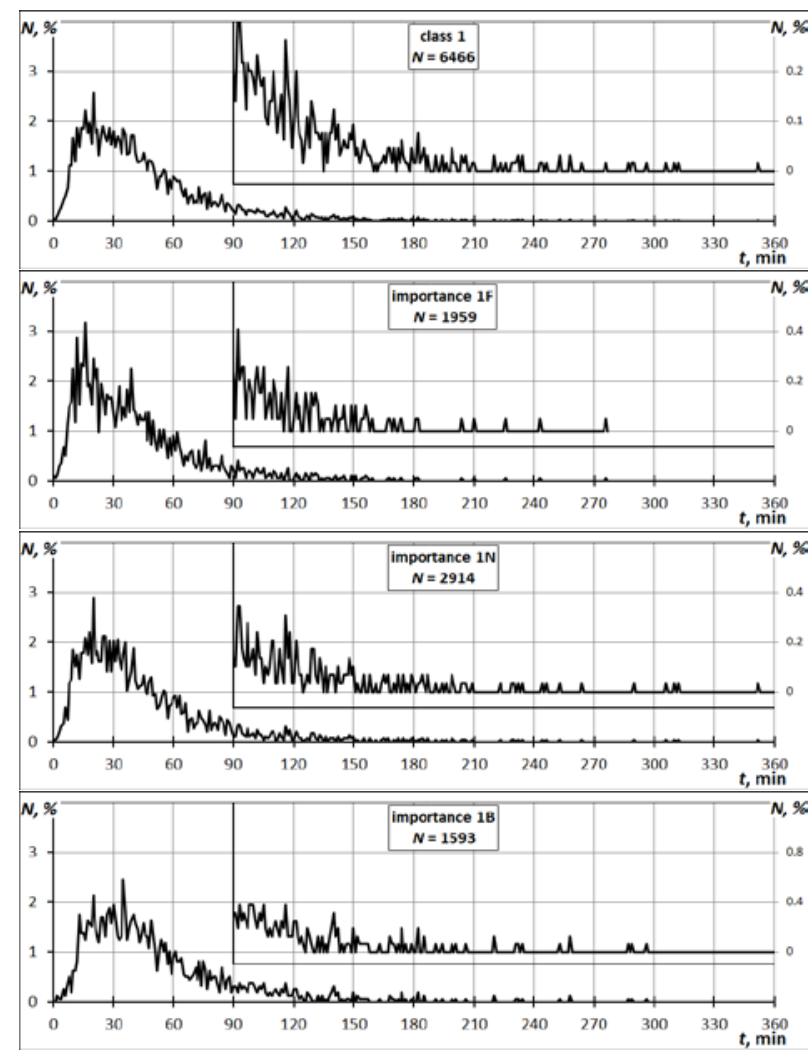

Figure 4. Duration distribution of class 1 flares
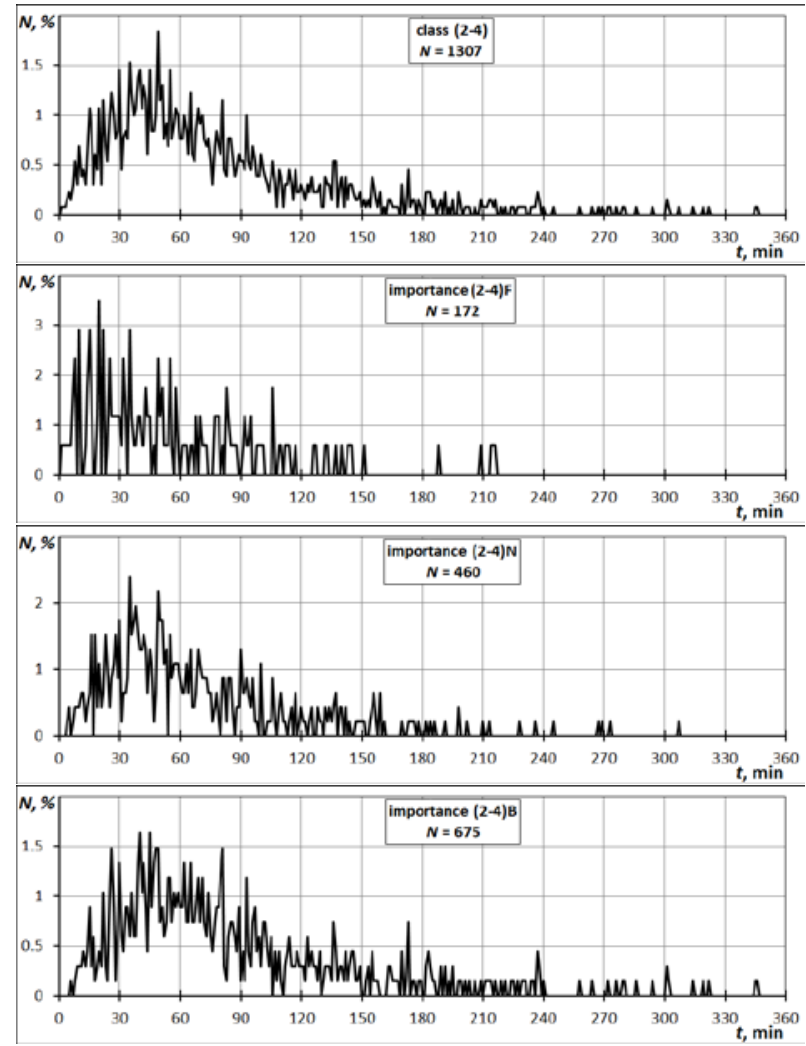

Figure 5. Duration distribution of class 2-4 flares

Confidence intervals are determined by the formula

$$
\bar{t}-\Omega(P, N-1) \frac{\sigma}{\sqrt{N}} \leq \alpha \leq \bar{t}+\Omega(P, N-1) \frac{\sigma}{\sqrt{N}},
$$

where $\bar{t}$ is the mean duration of flares, $\Omega$ is Student's coefficient (1.96), $P$ is the confidence coefficient (95\%), $N$ is the number of flares.

Since with increasing class of area the distributions begin to exhibit a multipeak structure, associated apparently with a decrease in the number of flares, we estimate modal times from smoothed distribution curves.

Figure 6 shows variations in statistical parameters of distributions with increasing class of area.

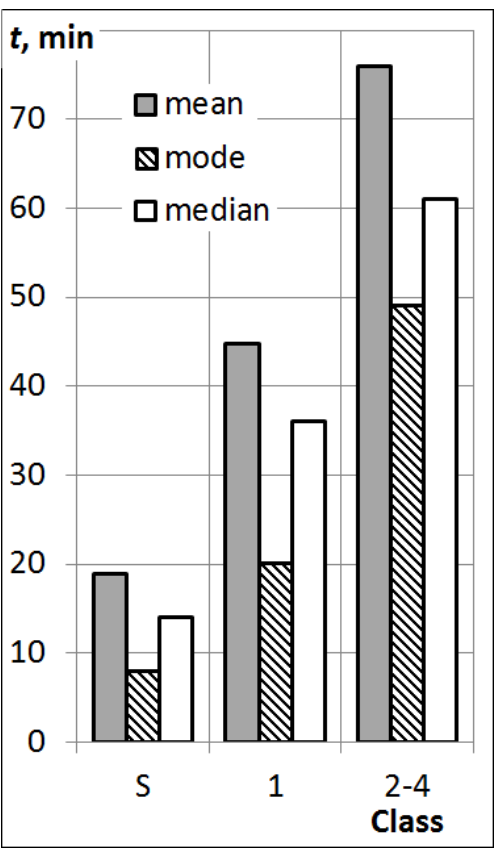

Figure 6. Variations in statistical distribution parameters with increasing class of area

The results indicate that with increasing importance and class of area of flares their mean duration increases from 17.4 to $85.5 \mathrm{~min}$; the mode shifts from 8 to 49 min; the median, from 13 to $69 \mathrm{~min}$. Time intervals for $90 \%$ of flares also increase.

Statistical parameters of flares by brightness are given in Table 3. No longitude restrictions are imposed in this case. By comparison, the next-to-last column of Table 3 lists median times obtained by Temmer et al. [2001].

Table 3

\begin{tabular}{|c|c|c|r|r|l|l|}
\hline Brightness & $N$ & $\bar{t} \pm \alpha$ & $M o$ & $M e$ & $M e^{\mathrm{T}}$ & $\Delta t$ \\
\hline F & 70390 & $17.8 \pm 0.1$ & 8 & 13 & 13 & $\begin{array}{l}1- \\
36\end{array}$ \\
\hline N & 26697 & $25.3 \pm 0.3$ & 10 & 18 & 19 & $\begin{array}{l}1- \\
51\end{array}$ \\
\hline B & 7210 & $37.3 \pm 0.9$ & 12 & 26 & 24 & $\begin{array}{l}1- \\
79\end{array}$ \\
\hline$\Sigma$ & 104297 & $21.1 \pm 0.1$ & 8 & 15 & 15 & $1-$ \\
& & & & & & 43 \\
\hline
\end{tabular}

There is a clear relationship between an increase in duration of flares and an increase in class of brightness (Table 3). One of the conclusions drawn by Borovik, Zhdanov [2017b] is confirmed: with increasing optical importance, the number of flares with a higher brightness class increases (Table 3). 


\section{DURATION OF FLARES AS DERIVED FROM HOLL, LEAR, RAMY DATA}

To verify the reliability of the results, we have carried out a similar analysis, using data from Holloman Solar Observatory, USA (HOLL), Learmonth Solar Observatory, Australia (LEAR), and Ramey Solar Observatory, Puerto Rico (RAMY). The number of flares is given in Table 4, distribution parameters are shown in Figures 7 and 8.

According to data from the three observatories, the mean duration of low-power flares varies insignificantly (Figures 7, 8, a).

Table 4

\begin{tabular}{|l|r|r|r|r|}
\hline & $\begin{array}{c}\text { All sta- } \\
\text { tions }\end{array}$ & HOLL & LEAR & RAMY \\
\hline SF & 55799 & 14232 & 11838 & 8353 \\
\hline SN & 18538 & 2570 & 1643 & 1976 \\
\hline SB & 3867 & 974 & 343 & 841 \\
\hline $1 \mathrm{~F}$ & 1959 & 326 & 455 & 175 \\
\hline $1 \mathrm{~N}$ & 2912 & 421 & 447 & 199 \\
\hline $1 \mathrm{~B}$ & 1590 & 369 & 251 & 200 \\
\hline$(2-4) \mathrm{F}$ & 172 & 28 & 35 & 9 \\
\hline$(2-4) \mathrm{N}$ & 460 & 60 & 78 & 29 \\
\hline$(2-4) \mathrm{B}$ & 671 & 164 & 155 & 84 \\
\hline S & 78204 & 17776 & 13824 & 11170 \\
\hline 1 & 6461 & 1116 & 1153 & 574 \\
\hline $2-4$ & 1303 & 252 & 268 & 122 \\
\hline$\sum$ & 85968 & 19144 & 15245 & 11866 \\
\hline
\end{tabular}

For flares of higher classes of area, they are within the dispersion interval $\bar{\sigma}$. The tendency for the mean duration to increase with increasing class of area is quite clear.

More significant differences occur in flare importance (Figure $8, b$ ). The reason is most likely to be the insufficient statistical weight of the data (especially for large flares). Nevertheless, the increase in flare duration with increasing importance is fairly well defined.

Summarizing the results, we can draw the following conclusions:

- Using extensive statistical data, we have obtained reliable values of the mean duration of solar flares of various classes of area and importance.

- We have confirmed that the mean duration of flares increases with increasing classes of area and brightness [Smith H., Smith E., 1966; Temmer et al., 2001]. A similar situation occurs when the flare importance increases.

\section{DURATION OF FLARES OF SOME TYPES}

There is a good tendency for the duration of flares to increase with increasing class of area, with distributions overlapping quite strongly (Figure 9,a). The interval $\Delta t=1-38 \mathrm{~min}$, which is typical for $90 \%$ of low-power flares, includes $53.8 \%$ of class 1 flares and $25.5 \%$ of large flares (Figure 9, $b$ ).
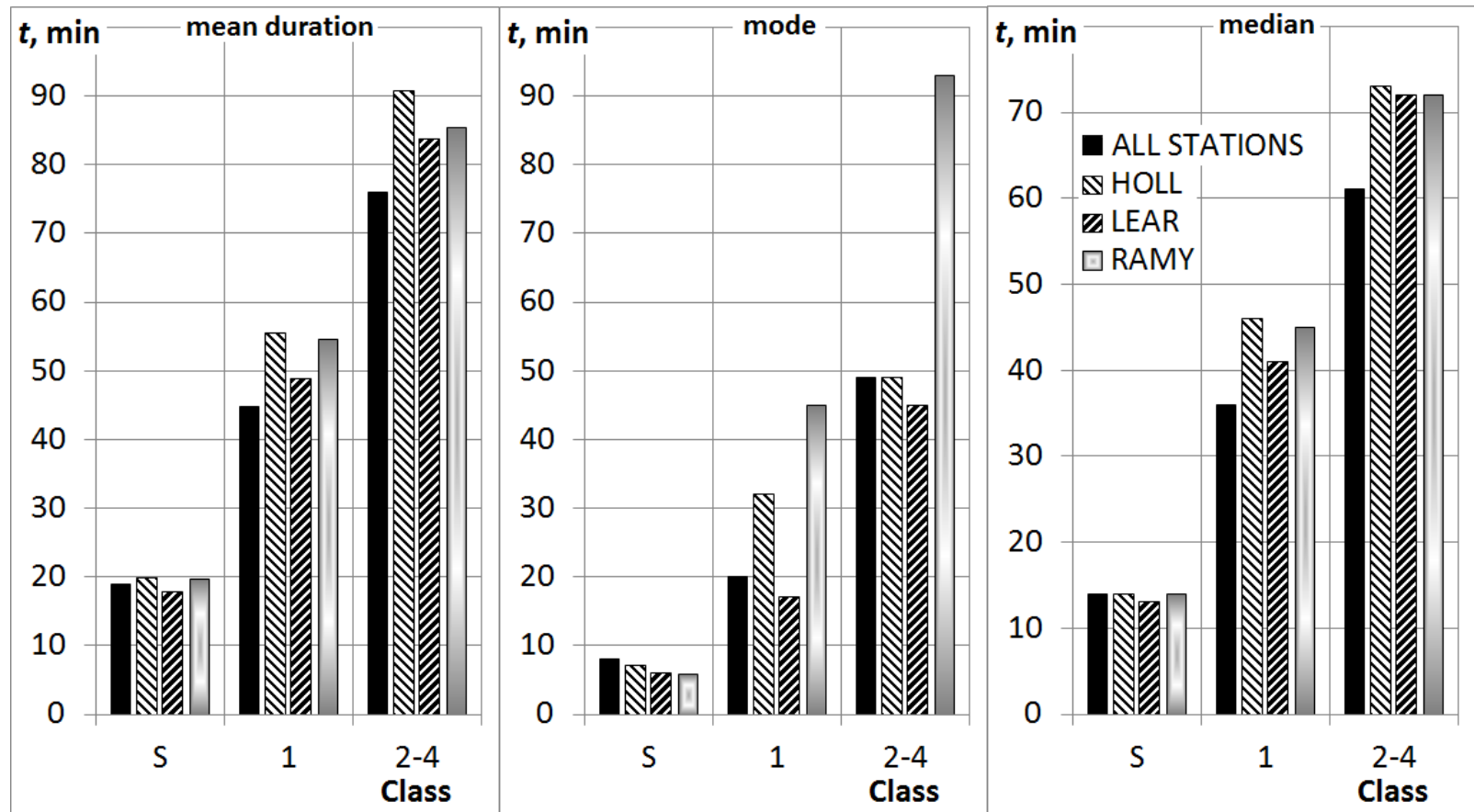

Figure 7. Variations in statistical parameters of distributions with increasing flare class of area as derived from HOLL, LEAR, and RAMY data 


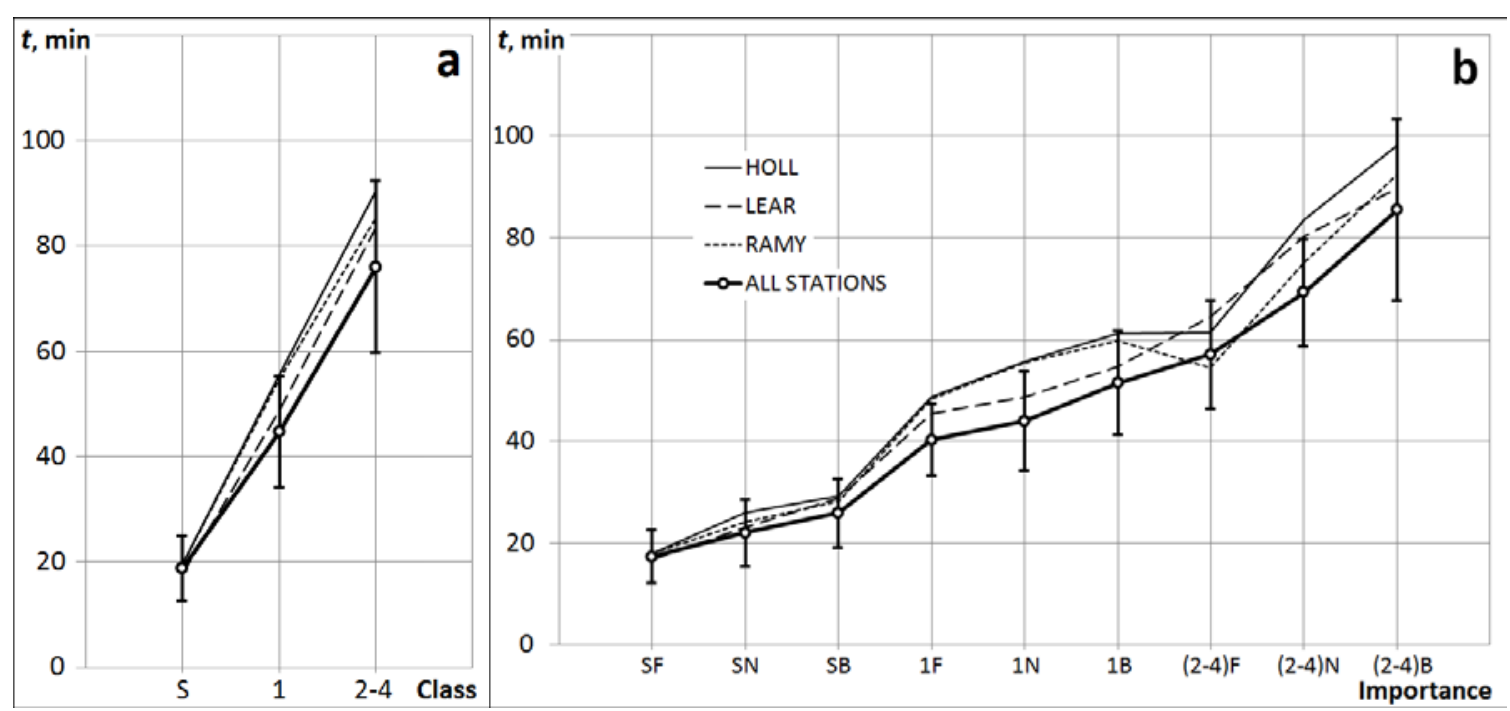

Figure 8. Variations in the mean duration of flares with increasing class of area $(a)$ and importance $(b)$ as derived from HOLL, LEAR, and RAMY data. Vertical lines show dispersion intervals $\bar{\sigma}$ (Figure 1)
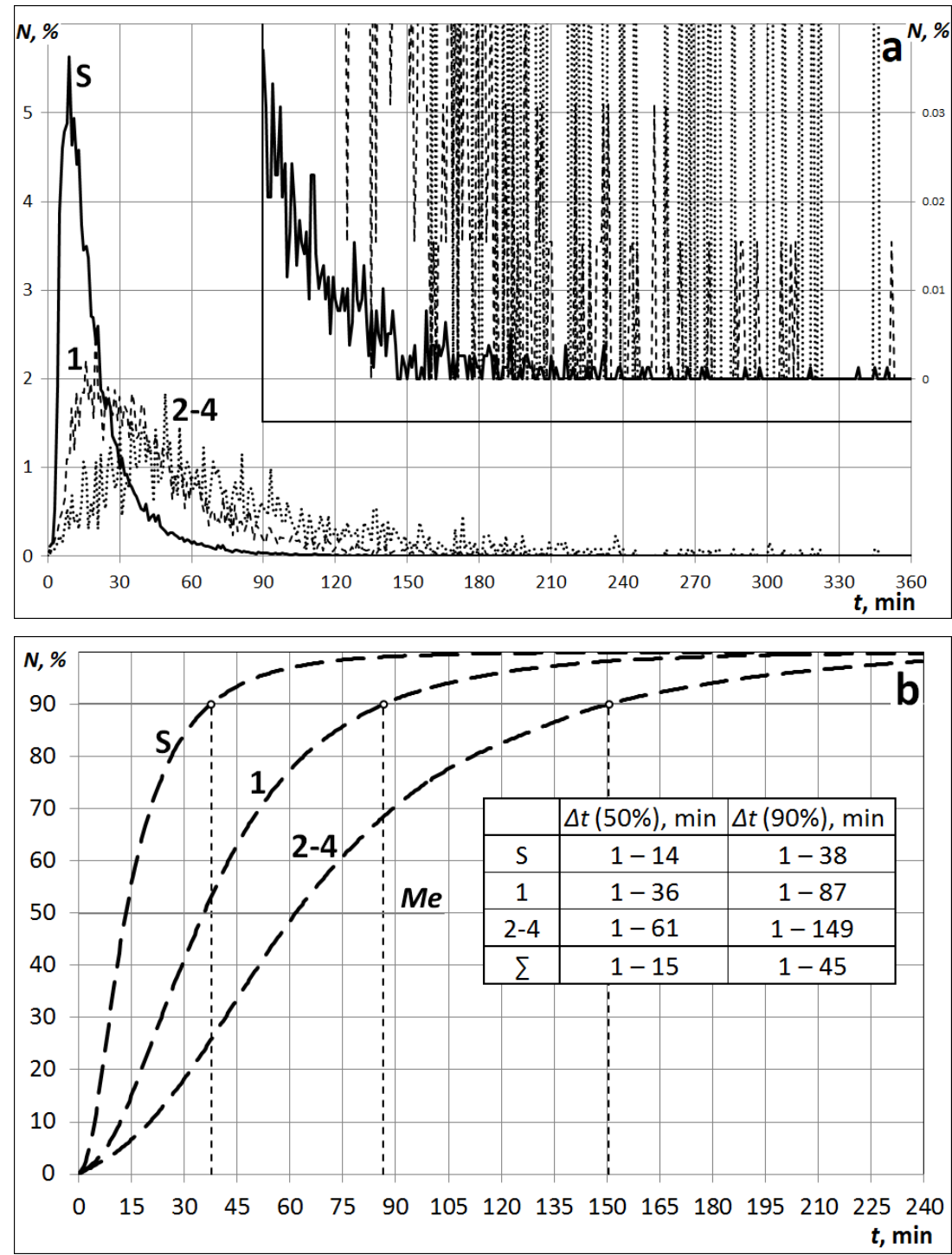

Figure 9. Duration distribution of class S, 1, and 2-4 flares: the solid line indicates class $\mathrm{S}$ flares; the dashed line, class 1 flares; the dotted line, class 2-4 flares (a). Curves of cumulative frequencies $(b)$. Table lists time intervals for 50 and $90 \%$ of flares 


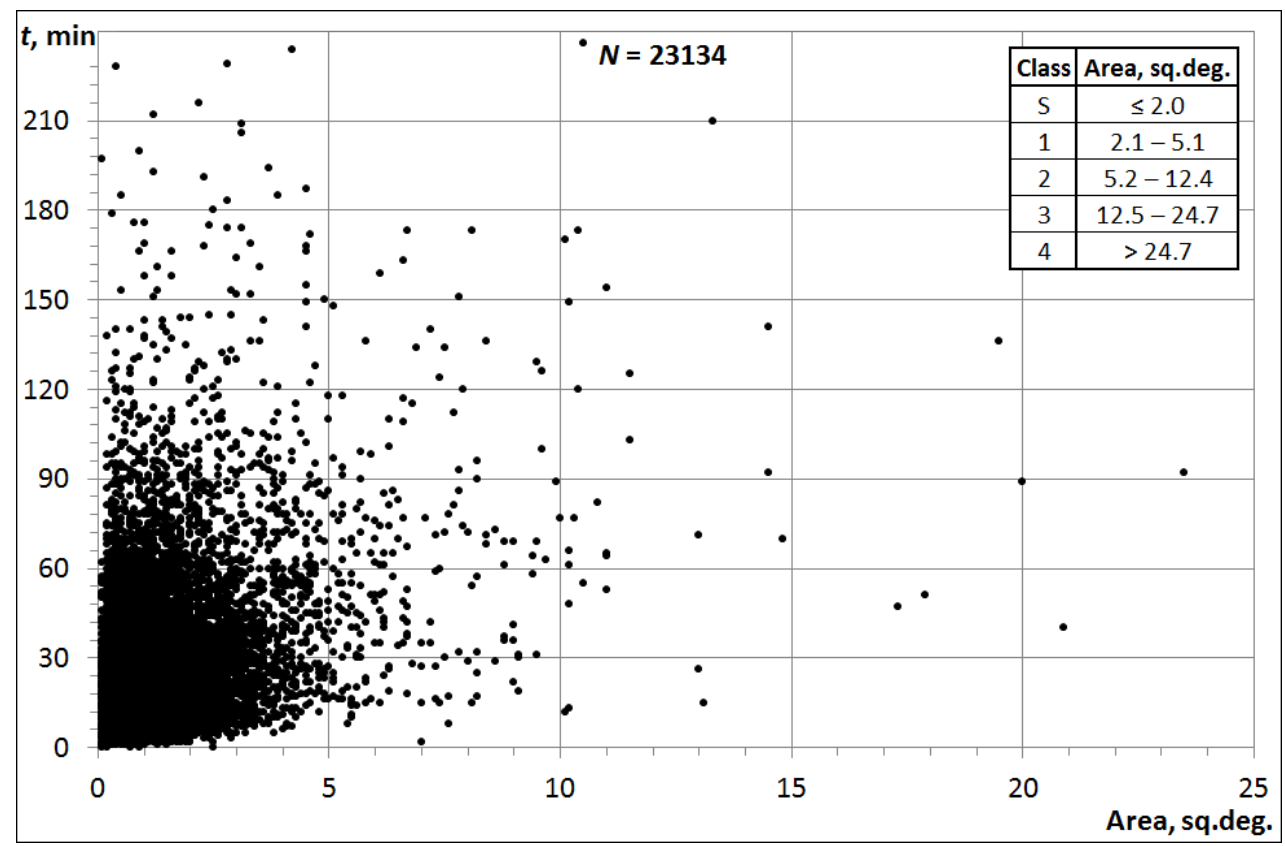

Figure 10. Flare duration as a function of area. Table shows the classification of solar flares in the $\mathrm{H} \alpha$ line

Figure 10 suggests that there is no direct functional relationship between duration and area of flares. The correlation coefficient does not exceed 0.3 .

To find the cause for the wide range of lifetimes of solar flares, we have conducted a similar statistical analysis for flares of individual types.

The international solar patrol has identified 26 distinctive features of flare development. They generally represent the flare type, its related events, area of origin, size of active region, etc. Below is the list of flare development features which we consider the most important:

- D - Brilliant point;

- E - Two or more brilliant points;

- G - No visible spots nearby;

- $\mathrm{H}$ - Flare accompanied by high-speed dark filament;

- $\mathrm{K}$ - Several intensity maxima;

- L - Existing filaments suddenly active;

- M - White-light flare;

- $\mathrm{R}$ - Asymmetry in $\mathrm{H} \alpha$ line suggests high-speed mass ejection;

- S - Brightness followed filament disappearance in the same position;

- U - Two bright branches, parallel or converging;

- V - An explosive phase: important expansion in about 1 min that often includes a significant intensity increase;

- W - Great area increase after time of maximum brightness;

- Z - Major sunspot umbra covered by flare.

Unfortunately, not all stations observe features of flare development; therefore we have analyzed only flares of seven types that have a sufficiently large statistical weight. We impose the same restrictions on the data as for all flares. The statistical parameters obtained for flares of these types are given in Table 5 and in Figure 11.
By comparison, the last row of Table 5 shows parameters for all flares. The confidence intervals for large flares being quite wide, some of the conclusions will be based on the data on class $\mathrm{S}$ and 1 flares.

The results suggest that the flare types considered differ greatly in duration.

Among flares of all types, flares with one brilliant point (D) have the shortest lifetime: for classes $\mathrm{S}$ and 1 $\bar{t}=15$ and $28 \mathrm{~min}$, which is approximately 1.5 times less than for flares with two or more brilliant points (E). Borovik and Myachin [2010] have established that such centers fade out slowly and live almost until the end of the flare. This suggests that the energy coming from a coronal source along magnetic tubes to the chromosphere is localized in those areas of the flare region where brightness is the highest. Švestka et al. [1982] have found out and Fletcher et al. [2011] have confirmed that the brightest flare areas are associated with footpoints of X-ray loops. It is natural to assume that the more channels connect a coronal source (or sources) with a chromospheric flare, the longer is its lifetime.

The longest-lived flares of those considered are flares whose light curves show several intensity maxima (K). Their lifetime ( $\bar{t}=46$ and $73 \mathrm{~min}$ ) is $2.4-$ 1.6 times longer than that of explosive $\mathrm{V}$-type flares ( $\bar{t}=19$ and $45 \mathrm{~min}$ ) and 3.0-2.6 times longer than that of D-type flares. The intermittent increase in flare brightness and the multiple intensity maxima are obviously related to the dynamics of the energy release region. The energy seems to be released gradually in different flare arches, and the energy release in an individual magnetic flux tube corresponds to each flare [Borovik et al., 2014]. 


\begin{tabular}{|c|c|c|c|c|c|c|c|c|c|c|c|c|c|c|c|}
\hline & \multicolumn{5}{|c|}{ class S } & \multicolumn{5}{|c|}{ class 1} & \multicolumn{5}{|c|}{ classes 2-4 } \\
\hline type & $N$ & $\overline{\bar{t}} \pm \alpha$ & Mo & $M e$ & $\Delta t$ & $N$ & $\overline{\bar{t}} \pm \alpha$ & $M o$ & $M e$ & $\Delta t$ & $N$ & $\overline{\bar{t}} \pm \alpha$ & $M o$ & $M e$ & $\Delta t$ \\
\hline $\mathrm{D}$ & 3445 & $15.2 \pm 0.4$ & 7 & 11 & $1-30$ & 227 & $27.7 \pm 3.4$ & 10 & 18 & $1-58$ & 31 & $65.1 \pm 17.5$ & 41 & 51 & $1-114$ \\
\hline$E$ & 7009 & $22.5 \pm 0.5$ & 10 & 17 & $1-45$ & 1527 & $40.9 \pm 1.5$ & 20 & 32 & $1-80$ & 234 & $68.6 \pm 6.3$ & 40 & 54 & $1-137$ \\
\hline $\mathrm{V}$ & 2943 & $19.1 \pm 0.5$ & 10 & 15 & $1-36$ & 286 & $44.7 \pm 4.2$ & 25 & 34 & $1-91$ & 53 & $83.2 \pm 15.5$ & 50 & 65 & $1-173$ \\
\hline $\mathrm{K}$ & 1034 & $46.0 \pm 2.1$ & 24 & 38 & $1-85$ & 309 & $72.8 \pm 5.6$ & 33 & 58 & $1-144$ & 98 & $97.2 \pm 10.9$ & 70 & 78 & $1-173$ \\
\hline $\mathrm{H}$ & 2370 & $19.3 \pm 0.7$ & 8 & 14 & $1-40$ & 436 & $38.8 \pm 2.6$ & 19 & 32 & $1-74$ & 128 & $65.0 \pm 7.2$ & 52 & 56 & $1-119$ \\
\hline $\mathrm{U}$ & 574 & $32.7 \pm 2.0$ & 8 & 26 & $1-64$ & 440 & $63.7 \pm 3.9$ & 31 & 55 & $1-118$ & 229 & $109.1 \pm 8.0$ & 87 & 93 & $1-199$ \\
\hline $\mathrm{G}$ & 498 & $21.7 \pm 1.5$ & 12 & 17 & $1-42$ & 73 & $37.3 \pm 8.1$ & 10 & 27 & $1-80$ & 6 & $71.8 \pm 29.1$ & 79 & 77 & $1-128$ \\
\hline & 78204 & $18.9 \pm 0.1$ & 8 & 14 & $1-38$ & 6461 & $44.7 \pm 0.8$ & 20 & 36 & $1-87$ & 1303 & $76.0 \pm 0.3$ & 49 & 61 & $1-149$ \\
\hline
\end{tabular}

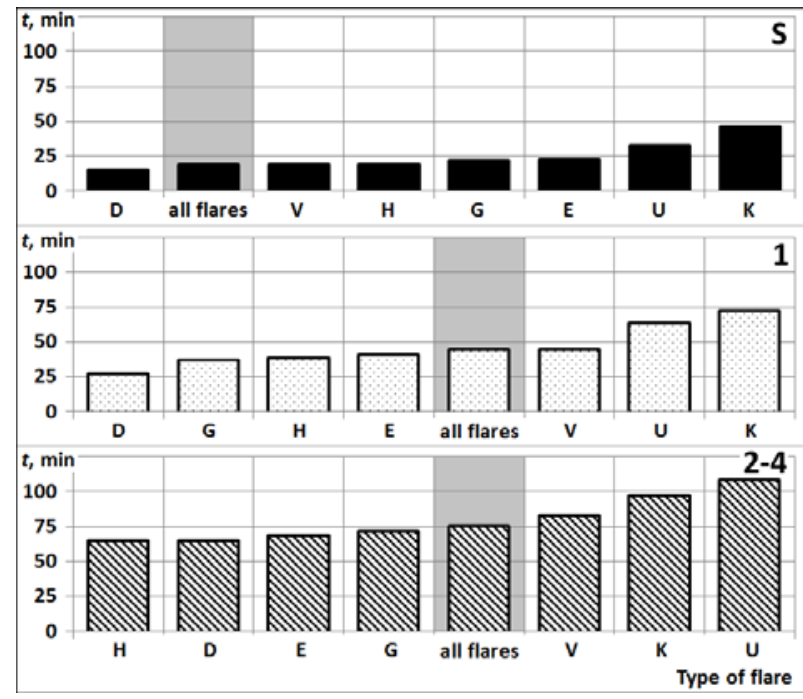

Figure 11. Mean duration of flares of individual types as a function of class of area. The histograms are plotted in ascending order of $\bar{t}$

Sometimes, flares of a certain type have several features. For example, explosive flares most often occur in places where S filaments disappear. Among them there are almost no flares with several intensity maxima (K). In a large number of cases, there is only one brilliant point (D) in the flare region. This suggests that the physical nature of such flares differs from, say, the nature of two-ribbon flares (U). Explosive flares are most likely to be related to the so-called Hyder flares, which, according to the gravity model [Hyder, 1967], arise due to the fall of the eruptive prominence matter from the corona to the chromosphere.

Two-ribbon flares in duration are in the second to last place in the time series of Figure 11 (before $\mathrm{K}$ flares). Their mean duration for class $S$ and 1 flares is 33 and 64 min, which is much longer than that for all flares. Two-ribbon flares generally have several brilliant points (E). A high percent of flares shows multiple intensity maxima (K). The mechanism of development of two-ribbon flares is represented by the CSHKP model [Carmichael, 1964; Sturrock, 1966; Hirayama, 1974; Kopp, Pneuman, 1976].

It is noteworthy that as class of area increases flares of some types change their relative positions on the time scale (Figure 11). For example, V flares move into the category of flares with longer duration. Flares with two or more brilliant points (E), on the contrary, go into the category of flares with shorter duration.

We can conclude that the spatial and temporal features of development largely determine the duration of flares and explain to some extent the wide range of lifetimes of solar flares. We can assume that since the number of flares of different types and classes of area vary with solar cycle phase, the total mean duration of solar flares also varies: decrease at solar minimum and increase at solar maximum.

\section{ULTRA-LONG SOLAR FLARES}

94.6\% of all flares considered lasted less than one hour, and $5.4 \%$ (6675 flares) lived much longer. Statistical parameters of ultra-long solar flares along with the range of durations (L) are shown in Table 6. For $90 \%$ of flares with $t>60 \mathrm{~min}$, the duration was $2.1-3.3 \mathrm{hrs}$.

Ultra-long flares can have almost any class of area, but most of them are low-power. For class $\mathrm{S}$ and 1 flares, the maximum duration was 9.2 and 11.7 hrs; for large flares, 8.3 hrs. In very rare cases, flares exist for about 12 hrs.

Table 6

\begin{tabular}{|l|r|c|c|c|c|c|}
\hline & \multicolumn{1}{|c|}{$N$} & $N, \%$ & $\bar{t} \pm \alpha$ & $\mathrm{Me}$ & $\Delta t$ & $L$ \\
\hline $\mathrm{S}$ & 3649 & 54.7 & $86.3 \pm 1.1$ & 75 & $60-124$ & $60-549$ \\
\hline 1 & 2167 & 32.5 & $95.8 \pm 1.9$ & 82 & $60-142$ & $60-700$ \\
\hline $2-4$ & 859 & 12.9 & $118.8 \pm 4.2$ & 97 & $60-198$ & $60-499$ \\
\hline$\sum$ & 6675 & 100 & $93.6 \pm 1.1$ & 79 & $60-140$ & $60-700$ \\
\hline
\end{tabular}

In conclusion, Table 7 lists mean durations of flares for different classes of area, obtained by different authors at different times. The Table is formed using data from [Smith H., Smith E., 1966; Temmer et al., 2001]; it is corrected and complemented. The top part of Table 7 presents flare classes of area introduced in 1956 (the first international classification of solar flares in the $\mathrm{H} \alpha$ line).

Table 8 shows the modal and median times of distributions.

The time parameters of solar flares we obtained in this study differ in most cases from those obtained by other authors. One of the reasons is most likely to be the insufficient number of data used by the authors for statistical analysis. This is confirmed, in particular, by the fact that the values of the mean duration of low-power flares we derived completely coincide with those obtained by Temmer et al. [2001]. 


\begin{tabular}{|c|c|c|c|c|c|c|c|c|c|}
\hline \multirow[b]{3}{*}{ Authors } & \multirow[b]{3}{*}{ Period } & \multirow{3}{*}{$\begin{array}{l}\text { Number } \\
\text { of flares }\end{array}$} & \multicolumn{7}{|c|}{ Mean duration } \\
\hline & & & $1-$ & 1 & 2 & 3 & $3+$ & $2,3,3+$ & \multirow[b]{2}{*}{$\sum$} \\
\hline & & & $\mathrm{S}$ & 1 & 2 & 3 & 4 & $2-4$ & \\
\hline Newton \& Barton (1937) ${ }^{1}$ & $1935-1936$ & - & - & - & - & - & - & - & $20.0-40.0$ \\
\hline Waldmeier (1938) & 1935-1937 & 357 & - & 21.0 & 38.0 & 61.0 & - & - & 27.0 \\
\hline Giovanelli (1948) & 1937-1938 & 24 & - & - & - & - & - & - & 30.0 \\
\hline Waldmeier (1948) & $1935-1944$ & 927 & - & 20.3 & 33.4 & 62.4 & - & - & 24.8 \\
\hline Ellison (1949) & 1935-1947 & 109 & - & 17.0 & 29.0 & 62.0 & $\begin{array}{c}\approx 180 \\
0\end{array}$ & - & - \\
\hline Warwick (1954) & 1951-1953 & 357 & 31.0 & \multicolumn{4}{|c|}{60.0} & - & 40.0 \\
\hline Dodson et al. $(1956)^{2}$ & 1949-1952 & 194 & 28.0 & 43.0 & 66.0 & \multicolumn{2}{|c|}{84.0} & - & - \\
\hline $\begin{array}{l}\text { Waldmeier \& } \\
\text { (1959) }\end{array}$ & 1945-1954 & 1604 & - & 22.1 & 44.8 & 84.9 & - & - & 28.1 \\
\hline H. Smith and E. Smith (1966) & 1957-1958 & - & 16.8 & 32.2 & 68.8 & 146.0 & - & - & 39.4 \\
\hline Reid (1968) & 1958-1965 & 2907 & 16.5 & 28.2 & - & - & - & 60.5 & - \\
\hline Ružičková-Topolová (1974) ${ }^{4}$ & $1957-1965$ & 661 & - & - & 71.3 & 129.9 & 305.5 & - & - \\
\hline Wilson (1983) & 1980 & 1348 & - & - & - & - & - & - & 29.8 \\
\hline Antalova (1985) & 1970-1974 & 460 & 27.0 & 55.0 & 78.0 & - & - & 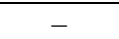 & - \\
\hline & 1975-1979 & 561 & 30.0 & 51.0 & 78.0 & 201.0 & - & - & - \\
\hline Wilson (1987) & 1975 & 850 & 16.6 & 38.7 & 62.7 & - & - & - & 18.1 \\
\hline Barlas \& Altas (1992) ${ }^{5}$ & 1947-1990 & 3569 & 24.0 & 38.0 & - & - & - & 78.0 & - \\
\hline This paper ${ }^{5}$ & $1972-2010$ & 577 & 21.7 & 37.3 & - & - & - & 71.8 & 24.2 \\
\hline Temmer et al. (2001) & 1975-1999 & 75739 & 18.9 & 35.7 & 66.3 & 116.0 & - & - & 20.6 \\
\hline This paper & $1972-2010$ & 85968 & 18.9 & 44.7 & 72.8 & 106.9 & 85.6 & 76.0 & 21.7 \\
\hline
\end{tabular}

${ }^{1}$ The smaller number refers to less intense flares; the larger number, to more intense ones.

${ }^{2}$ The duration is calculated from photometric curves of flares.

${ }^{3}$ The data have been obtained from the results of the International Geophysical Year (IGY).

${ }^{4}$ Only large solar flares are considered.

${ }^{5}$ Only spotless solar flares are considered.

Table 8

\begin{tabular}{|c|c|c|c|c|c|c|c|c|c|c|c|c|c|}
\hline \multirow{3}{*}{ Authors } & \multirow{3}{*}{ Period } & \multicolumn{6}{|c|}{ Mode } & \multicolumn{6}{|c|}{ Median } \\
\hline & & $1-$ & 1 & 2 & 3 & $2,3,3+$ & \multirow[t]{2}{*}{$\sum$} & $1-$ & 1 & 2 & 3 & $\begin{array}{c}2,3, \\
3+\end{array}$ & \multirow[t]{2}{*}{$\sum$} \\
\hline & & $\mathrm{S}$ & 1 & 2 & 3 & $2-4$ & & $\mathrm{~S}$ & 1 & 2 & 3 & $2-4$ & \\
\hline \multirow[t]{3}{*}{$\begin{array}{l}\text { H. Smith } \\
\text { and E. Smith (1966) }\end{array}$} & 1935-1944 & - & 7.5 & 22.5 & 57.5 & - & 7.5 & - & 13.0 & 22.5 & 70.0 & - & 15.0 \\
\hline & 1945-1954 & - & 7.5 & 22.5 & 50.0 & - & 7.5 & - & 16.0 & 33.0 & 40.0 & - & 23.5 \\
\hline & 1957-1958 & 10 & 12.5 & 62.5 & 62.5 & - & 25.0 & 13 & 23.5 & 55.0 & 130.0 & - & 25.0 \\
\hline Temmer et al. (2001) & 1975-1999 & - & - & - & - & - & 8 & 14 & 28 & - & - & 57 & 15 \\
\hline This paper & 1972-2010 & 8 & 20 & 49 & 71 & 49 & 8 & 14 & 36 & 59 & 86 & 61 & 15 \\
\hline
\end{tabular}

\section{CONCLUSIONS}

1. From extensive statistical material, we have first obtained the most complete and reliable data on mean duration of solar flares of different importance and classes of area. We have confirmed the tendency for the mean duration of flares to increase with increasing classes of area and brightness [Smith H., Smith E., 1966; Temmer et al., 2001]. A similar tendency has been revealed for flare importance.

2. We have shown that despite the well-pronounced tendency, distributions of flares over time substantially overlap; there is no direct functional relationship between duration and area of flares.

3. We have established that the duration of flares depends on features of their development. We have first obtained values of the mean duration for flares of seven types. We have established that among flares of the types considered, the flares with the one brilliant point in the flare region have the shortest mean duration, the flares with several intensity maxima and two-ribbon flares have the longest mean duration. Flares of all types have a tendency for their duration to increase with increasing class of area.

4. We have found that $94.6 \%$ of solar flares last less than $1 \mathrm{hr}, 90 \%$ of ultra-long flares ( $t>60 \mathrm{~min}$ ) exist for 2.1-3.3 hrs. In very rare cases, flares can exist for $12 \mathrm{hrs}$. For large solar flares, the maximum duration is less than $8.3 \mathrm{hrs} ; 54.7 \%$ of ultra-long flares are low-power.

The work was performed with budgetary funding of the Basic Research Program II.16.

\section{REFERENCES}

Altyntsev A.T., Banin V.G., Kuklin G.V., Tomozov V.M. Solnechnye vspyshki [Solar Flares]. Moscow, Nauka Publ., 1982, 246 p. (In Russian).

Antalova A. Comprehensive solar flare indices and duration of $\mathrm{H}$-alpha flares. Contributions of the Astronomical Observatory Skalnate Pleso. 1985, vol. 13, pp. 243-246.

Barlas O., Altas L. The duration of spotless flares. Astrophys. Space Sci. 1992, vol. 197, no. 2, pp. 337-341. DOI: 10.1007/BF00645745. 
Borovik A.V., Myachin D.Yu. Structure and development of the spotless flare on March 16, 1981. Geomagnetism and Aeronomy. 2010, vol. 50, no. 8, pp. 937-949. DOI: 10.1134/S0016793210080037.

Borovik A.V., Zhdanov A.A. Statistical studies of lowpower solar flares. Main phase duration. Solar-Terrestrial Physics. 2017a, vol. 3, no. 4, pp. 5-16. DOI: 10.12737/stp34201701.

Borovik A.V., Zhdanov A.A. Statistical studies of lowpower solar flares. Distributions of flares by area, brightness and classes. Solar-Terrestrial Physics. 2017b, vol. 3, no. 1, pp. 40-56. DOI: 10.12737/article_58f96fda7e3e76.83058648.

Borovik A.V., Myachin D.Yu., Tomozov V.M. Observation of spotless solar flares at Baikal Astrophysical Observatory of ISTP SB RAS and their interpretation. Izvestiya Irkutskogo Gosudarstvennogo Universiteta» Ser. "Nauki o Zemle" [The Bulletin of Irkutsk State University. Ser. Earth Sciences]. 2014, vol. 7, no. 1, pp. 23-45. (In Russian).

Carmichael H. A process for flares. Proc. AAS-NASA Symp. on the Physics of Solar Flares (NASA-SP 50). 1964, pp. 451-456.

Dodson H.W., Hedeman E.R., McMath R.R. Photometry of solar flares. Astrophys. J. Suppl. 1956, vol. 2, pp. 241-270.

Ellison M.A. Characteristic properties of chromospheric flares. Monthly Not. Royal Astron. Soc. 1949, vol. 109, pp. 3-27.

Fletcher L., Dennis B.R., Hudson H.S., Krucker S., Phillips K., Veronig A., Battaglia M., Bone L.. Caspi A., Chen Q., Gallagher P., Grigis P.T., Ji H., Liu W., Milligan R.O., Temmer M. An observational overview of solar flares. Space Sci. Rev. 2011, vol. 159, pp. 19-106. DOI: 10.1007/s11214-0109701-8.

Giersch O. GONG Inter-site H $\alpha$ flare comparison. J. Phys. Conf. Ser. 2013, vol. 440, 0120062013. DOI: 10.1088/17426596/440/1/012006.

Giovanelli R.G. Chromospheric flares. Monthly Not. Royal Astron. Soc. 1948, vol. 108, pp.163-176.

Hirayama T. Theoretical model of flares and prominences. I: evaporating flare model. Solar Phys. 1974, vol. 34, no. 2, pp. 323-338.

Hyder C.L. A phenomenological model for disparitions brusques followed by flare-like chromospheric brightenings. Solar Phys. 1967, vol. 2, no. 3, pp. 267-284.
Kopp R.A., Pneuman G.W. Magnetic reconnection in the corona and the loop prominence phenomenon. Solar Phys. 1976, vol. 50, pp. 85-98.

Newton H.W., Barton H.J. Bright solar eruptions and radio fadings during the years 1935-1936. Monthly Not. Royal Astron. Soc. 1937, vol. 97, no. 8, pp. 594-611.

Potzi W., Veronig A.M., Riegler G., Amerstorfer U., Pock T., Temmer M., Polanec W. Real-time flare detection in groundbased $\mathrm{H} \alpha$ imaging at Kanzelhöhe Observatory. Solar Phys. 2014, vol. 290, no. 3, pp. 951-977.

Quarterly Bulletin on Solar Activity. 1972-1973.

Reid J.H. Cape Lyot H $\alpha$-heliograph results. Solar Phys. 1968, vol. 5, no. 2, pp. 207-235.

Ružičková-Topolová B. Some characteristics of great solar flares in the period 1957-1965. Bull. Astron. Inst. Czechosl. 1974, vol. 25, pp. 345-352.

Solar Geophysical Data. 1975-2010.

Smith H., Smith E. Solnechnye vspyshki [Solar Flares] Moscow, Mir Publ., 1966, 426 p. (In Russian). English edition: Smith H.J., Smith E. Solar Flares. Macmillan, 1963, 322 p.

Sturrock P.A. Model of the high-energy phase of solar flares. Nature. 1966, vol. 211, pp. 695-697.

Švestka Z., Dodson-Prince H.W., Martin S.F., Mohler O.C., Moore R.L., Nolte J.T., Petrasso R.D. Study of the postflare loops on 29 July 1973. Solar Phys. 1982, vol. 78, no. 2, pp. 271-285. DOI: 10.1007/BF00151609.

Temmer M., Veronig A., Hanslmeier A., Otruba W., Messerotti M. Statistical analysis of solar $\mathrm{H} \alpha$ flares. Astron. Astrophys. 2001, vol. 375, pp. 1049-1061.

Waldmeier M. Chromosphärische eruptionen. I. Mit 6 Abbildungen. Zeitschrift für Astrophysik. 1938, vol. 16, pp. 276-290.

Waldmeier M. Astron. Mitt. Zürich. 1948, no. 153.

How to cite this article

A.V. Borovik, A.A. Zhdanov. Statistical studies of duration of lowpower solar flares. Solar-Terrestrial Physics. 2018, vol. 4, no. 2, pp. 8-16. DOI: $10.12737 /$ stp-42201803 